\title{
SEXUAL SELECTION DETERMINES PARENTAL CARE PATTERNS IN CICHLID FISHES
}

\author{
Alejandro Gonzalez-Voyer, ${ }^{1,2}$ John L. Fitzpatrick, ${ }^{3,4}$ and Niclas Kolm ${ }^{1,5}$ \\ ${ }^{1}$ Animal Ecology, Department of Ecology and Evolution, Evolutionary Biology Centre, Uppsala University, Norbyvägen \\ 18D, 75236 Uppsala, Sweden \\ 2E-mail: alejandro.gonzalezvoyer@ebc.uu.se \\ ${ }^{3}$ Department of Biology, McMaster University, 1280 Main St. West, Hamilton, Ontario L8S 4K1, Canada \\ ${ }^{4}$ E-mail: fitzpajl@mcmaster.ca \\ ${ }^{5}$ E-mail: niclas.kolm@ebc.uu.se
}

Received December 18, 2007

Accepted May 1, 2008

Despite a massive research effort, our understanding of why, in most vertebrates, males compete for mates and females care for offspring remains incomplete. Two alternative hypotheses have been proposed to explain the direction of causality between parental care and sexual selection. Traditionally, sexual selection has been explained as a consequence of relative parental investment, where the sex investing less will compete for the sex investing more. However, a more recent model suggests that parental care patterns result from sexual selection acting on one sex favoring mating competition and lower parental investment. Using species-level comparative analyses on Tanganyikan cichlid fishes we tested these alternative hypotheses employing a proxy of sexual selection based on mating system, sexual dichromatism, and dimorphism data. First, while controlling for female reproductive investment, we found that species with intense sexual selection were associated with female-only care whereas species with moderate sexual selection were associated with biparental care. Second, using contingency analyses, we found that, contrary to the traditional view, evolutionary changes in parental care type are dependent on the intensity of sexual selection. Hence, our results support the hypothesis that sexual selection determines parental care patterns in Tanganyikan cichlid fishes.

KEY WORDS: BayesTraits, biparental care, female investment, parental investment, phylogenetic contingency analysis, sex roles.

In a diversity of species parents prolong investment in their progeny following egg laying or birth. Parental care, defined here in its narrowest sense as care of eggs or young when they are detached from the female's body (sensu Clutton-Brock 1991), is generally viewed as involving a trade-off between increased offspring survival and loss of mating opportunities and/or decreased investment in future reproduction (Trivers 1972; reviewed in Clutton-Brock 1991). Once parental care has evolved, conflict between mates may arise concerning how much care, if any, each parent will invest in the offspring (Westneat and Sargent 1996; Barta et al. 2002).

Observed patterns of relative parental investment by the sexes have traditionally been explained as resulting from anisogamy: sexual differences in gamete size and rate of production lead to differences in potential rate of reproduction and intrasexual competition among members of the small gamete producing sex for access to members of the large gamete producing sex (Bateman 1948). Trivers (1972) extended the argument based on anisogamy to incorporate both adult sex ratio (Emlen and Oring 1977) and mating system, suggesting that relative parental investment governs the operation of sexual selection. We refer to this as the "parental investment hypothesis" (PIH, Thomas and Székely 2005). The $\mathrm{PIH}$ predicts that differences between the sexes in the amount of parental investment result in competition among members of the sex investing less for access to the sex investing more. The ensuing intrasexual competition for access to mating partners will select 
against parental care by the competing sex because any amount of time or energy devoted to offspring will tend to decrease an individual's chances of mating with other partners (Trivers 1972).

However, despite being one of the most cited pieces of work in evolutionary biology, shortcomings in the PIH have been identified (e.g., Kokko and Jennions 2008). For instance, one of its underlying assumptions is that any investment in mating competition is traded off against parental care (Trivers 1972). Hence, the PIH does not explain sexual differences in competition for mates in species in which males compete for mates even though they are responsible for parental care, as occurs in many fish species (Baylis 1981; Clutton-Brock and Parker 1992; Andersson 1994). Furthermore, a more recent model suggested there is a fundamental flaw in Triver's argument and proposes as an alternative hypothesis that parental care patterns can actually be a consequence rather than a cause of sexual selection (Queller 1997). Every offspring has a single mother and a single father, thus the total number of matings in which males and females engage cannot differ. Anisogamy does not generate an inherent bias toward care by one sex if both sexes have identical future prospects of reproduction, which is generally the case when the adult sex ratio is at unity (Queller 1997; Kokko and Jennions 2003, 2008). Natural selection favoring reduced paternal care can only act on the proportion of males that actually participate in breeding. Hence, selection will favor a reduction in male care when there are fewer males available to mate than females, a condition that can be fulfilled by sexual selection acting either through malemale competition or female choice (Wade 1979; Queller 1997). Increased variance in male mating success resulting from sexual selection will tend to increase the costs of caring to breeding males because of lost mating opportunities. Moreover, male-male competition and multiple mating by females will tend to decrease male average paternity, further increasing the costs of caring for males (Queller 1997). In light of this argument the "sexual selection hypothesis" (SSH) suggests that rather than differences between the sexes in parental investment influencing sexual selection as suggested by Trivers (1972), the direction of causality could be reversed, anisogamy generates the conditions for sexual selection, which leads to postmating differences in parental care (Wade 1979; Queller 1997; Kokko and Jennions 2003, 2008).

Analyses of the PIH and SSH have been virtually limited to modeling approaches (e.g., Wade 1979; Clutton-Brock and Parker 1992; Parker and Simmons 1996; Queller 1997; Wade and Schuster 2002; Houston and McNamara 2005; Fromhage et al. 2007) and there is an important shortage of empirical data. Previous empirical studies have shown a correlation between the intensity of sexual selection and the relative parental investment of the sexes, and some suggest that sexual selection potentially influences parental care patterns (e.g., Reynolds and Székely 1997; Balshine-Earn and Earn 1998; Tallamy 2000; Ah-King et al. 2005;
Mank et al. 2005). Only two studies have analyzed the directionality of causation between parental care and sexual selection. Thomas and Székely (2005) analyzed the directionality of causation between parental care and sexual selection in shorebirds (Charadriiformes), but these authors compared the PIH with the influence of sexual conflict between partners on mating systems and did not analyze the alternative SSH. Mank et al.'s (2005) family-level analysis across 224 fish families did not detect any significant pattern of causation between parental care and sexual selection. However, this analysis may have lacked in power because it did not consider the rather substantial within-family variation in parental care and sexual selection in fish.

Modern comparative methods not only allow for tests of correlated evolution between traits while controlling for shared ancestry but also to analyse patterns of evolution in discrete binary traits and test whether evolutionary changes in one trait tend to be dependent on the state of a second trait, thus allowing inferences regarding the probable sequence of evolution of the traits of interest (Harvey and Pagel 1991; Pagel 1994, 1999). Such contingency methods are ideal for the analysis of the direction of causality between parental care and sexual selection when applied in a clade that presents variation in both the pattern of parental care and intensity of sexual selection, such as cichlid fishes. Tanganyikan cichlids present both uni- and biparental care and are highly diverse in other life-history traits (e.g., Goodwin et al. 1998; Konings 1998; Kolm et al. 2006). In this study we first tested the evolutionary correlation between intensity of sexual selection and parental care in Tanganyikan cichlids. Because female reproductive investment could potentially have an influence on whether males share the burden of parental care, for example possibly increasing the benefit of care for males, we also included clutch size in the analyses and tested whether it was correlated with parental care or sexual selection. Second, we analyzed, to our knowledge for the first time, the sequence of evolutionary transitions in care type and intensity of sexual selection using a Bayesian-based approach for contingency analyses (Pagel 1994, 1999; Pagel and Meade 2006) to test the PIH and SSH. We predicted that if the $\mathrm{PIH}$ is supported by our data, evolutionary transitions in the intensity of sexual selection will be contingent on parental care type. Alternatively, if the SSH is supported, transitions in parental care type will be contingent on the intensity of sexual selection.

\section{Methods DATA}

We collected data on sexual size dimorphism (SSD), sexual dichromatism, mating system, parental care, clutch size, and care type for 36 Tanganyikan cichlid species (see online Supplementary material). The species for which we managed to collect data provide a good reflection of the group's variation in these traits 
and represent most of the existing variety of ecological niches exploited by the Tanganyikan cichlids (Konings 1988, 2005; see online Supplemental material). Furthermore, they include species from 6 of the 12 tribes into which the Tanganyikan cichlids have been grouped (Salzburger et al. 2002).

We used combined information on mating system, sexual dichromatism, and SSD to estimate the intensity of sexual selection. We assumed sexual dichromatism and SSD both reflected sexual selection acting on males. Sexual dichromatism has been shown to be evolutionarily correlated with mating system (Seehausen et al. 1999) consistent with the hypothesis that it evolved as a result of sexual selection. "The magnitude and the direction of SSD in fish appear to be strongly related to the breeding system. Male-biased SSD tends to be associated with contest competition among males to defend resources or females (...)" (Fairbairn 2007, p. 6-7). SSD, sexual dichromatism, and mating system have previously been successfully used as proxies for sexual selection in comparative studies (e.g., Thomas and Székely 2005; Mank et al. 2005; Kolm et al. 2007), and sexual dichromatism has been suggested to be the most common manifestation of sexual selection in fish (Mank et al. 2005).

We measured standard length (SL) from sexed, wild-caught, sexually mature specimens of 33 species. For 18 of the 33 cichlid species we had SL data for several males and several females (range: 2-5 of each sex), for seven species we had data on more than one male (range: $2-5$ ) but only a single female, for the remaining eight species we had data on several females (range: 2-6) but only one male (see online Supplementary material). We are not aware of any evidence of spatial sexual segregation in cichlid fish (Barlow 2000) and we assumed that randomly captured specimens were representative of the population. We also complemented our data with SL measures for both sexes obtained from the published literature (e.g., Erlandsson and Ribbink 1997; Gashagaza 1991; see online Supplementary material). SSD estimated from our data was concordant with SSD estimated from data in the literature for 11 of 16 species. For the five species in which we had contrasting information we based our SSD estimate on the published data because the sample size was higher.

Data on mating system, sexual dichromatism, parental care type (female only or biparental), clutch size, and form of care (substrate guarding or mouthbrooding) were collected from primary publications and reference books (see online Supplementary material). Mating system in Tanganyikan cichlids can be divided into three broad categories: (1) monogamous where the durable pairbond constrains the number of females with which an attractive or competitive male can mate, (2) territorial and nonterritorial polygyny where constraints on the number of matings are relaxed as more attractive or competitive males can monopolize several females, and (3) promiscuity where males lek or otherwise defend territories that are visited by several females, and females may have their clutch fertilized by more than one male (Kuwamura 1997; Seehausen et al. 1999). Because our analyses require that all variables are coded as dichotomous (see below), all continuous or categorical variables were transformed into dichotomous variables. We coded mating system as a binary variable to represent the expected strength of sexual selection acting on males: a monogamous mating system was classified as presenting moderate intensity of sexual selection and all other mating systems were classified as presenting intense sexual selection. In Lepidolamprologus attenuatus, L. elongatus, and Neolamprologus brichardi bigamy is prevalent (35-50\% of observed males were bigamous; Gashagaza 1991) and in these cases mating system was classified as presenting intense sexual selection. Species were also coded as sexually dichromatic or nonsexually dichromatic based on information in the literature and color images in guidebooks (see online Supplementary material). Species were coded as sexually dichromatic if there were obvious differences in nonmelanic coloration between the sexes (Seehausen et al. 1999). We found data on mating system for 34 of the 36 species and on sexual dichromatism for 33 of the 36 species and only one species had missing data for both (see online Supplementary material). To be able to combine the information on sexual dichromatism, mating system, and SSD to estimate sexual selection we first transformed our measures of SSD into a dichotomous variable. SSD was coded as more dimorphic (when the male was $>5 \%$ longer than the female) or less dimorphic (male $<5 \%$ longer than the female). If SSD was dichotomized using a more conservative cut-off point (more dimorphic $=$ males $>10 \%$ larger than females, less dimorphic $=$ difference $<10 \%$ ) there was no change in the coding of sexual selection based on the composite measure (see below).

Species were coded as presenting either intense or moderate sexual selection based on our three proxies of sexual selection. To be coded as presenting intense sexual selection a species had to present any combination of at least two of the following: high SSD, sexual dichromatism, or nonmonogamous mating system. Alternatively, to be coded as presenting moderate sexual selection a species had to present any combination of at least two of the following: low SSD, absence of sexual dichromatism, or monogamous mating system. Three species (Eretmodus cyanosticus, Xenotilapia flavipinnis, X. longispinnis) were monogamous but showed pronounced SSD (males $>10 \%$ larger than females). In E. cyanosticus larger male size appears to result from female choice as larger females preferentially pair with larger males (B. Taborsky, pers. comm.). Size differences between males and females within pairs increase with female size and average differences in SL between sexes within pairs is about 12\% (B. Taborsky, pers. comm.). In X. flavipinnis and X. longispinnis males are more than 10\% larger than females (Erlandsson and Ribbink 1997), an SSD comparable to that in species with polygynous mating systems. Furthermore, mating system might not be defined 
independently of parental care patterns as shown by the following statement: "species which conduct biparental care are regarded as monogamous" (Kuwamura 1997, p. 61). Hence, E. cyanosticus, $X$. flavipinnis, and $X$. longispinnis were classified as presenting intense sexual selection based on the pronounced SSD. Coding these three species as presenting missing data for sexual selection (in which case they are allowed to take on both possible states in the analysis, see below) did not affect the results.

Our different measures of sexual selection were valid because analyses showed that SSD and sexual dichromatism were both significantly evolutionarily correlated with mating system in the cichlid species for which we had samples of all three measures (Pagel 94 analysis (see below): log-likelihood differences between independent and dependent models of evolution $=10.8, P=0.03$ and 13.2, $P=0.01$, respectively). Data on mating system and dichromatism were not available for one species and for a second species we had no data on mating system and had contrasting data on SSD and sexual dichromatism thus it could not be assigned to either intense or moderate sexual selection. These species were excluded from the Pagel 94 analyses involving sexual selection and treated as presenting missing data in the BayesTraits analysis (see below).

Parental care type was coded as a binary variable because all species presented either female only or biparental care. $\log _{10}$ clutch size was regressed on $\log _{10}$ female body length and we used the residuals as a size-corrected measure of female investment, because body length has been shown to be correlated with clutch size in cichlid fish (Kolm et al. 2006). Female investment was coded as high investment (if the residuals of clutch size vs. female body size $>0$ ) or low investment (residuals $<0$ ). Use of residuals to correct for a covariate has been criticized (Freckleton 2002), but here they were used to transform the variable into a dichotomous one and multiple regression was not applicable. Finally, form of care was also coded as a dichotomous variable representing either substrate guarding or mouthbrooding and was included in the analyses to control for potential confounding effects of form of care on sexual selection and female investment. It has been suggested that clutch size could be limited in mouthbrooding species by the number of eggs/fry that can fit into the mouth cavity and that biparental care in mouthbrooders occurs only in species with unusually large clutches (reviewed in Clutton-Brock 1991).

\section{PHYLOGENY}

We constructed an mtDNA phylogeny for the 36 Tanganyika cichlid species under study (Fig. 1). We used three mitochondrial genes, NADH 2 (1047 bp), cytochrome b (402 bp), and the more variable control region (369 bp), downloaded from GenBank (see online Supplementary material for sequence accession numbers) to reconstruct the phylogenetic relationships among species us- ing Bayesian analysis (Huelsenbeck et al. 2001) under a GTR plus $\Gamma$ model of sequence evolution (Salzburger et al. 2002). We specified Boulengerochromis microlepis and Bathybates fasciatus as the outgroup clade. A consensus tree was constructed using Bayesian analyses employing Metropolis coupled Markov chain Monte Carlo (MCMC) methods in MrBayes 3.1.1 (Ronquist and Huelsenbeck 2003). We ran two independent parallel analyses with four simultaneous chains in each for 3,000,000 iterations, sampling once every 100th iteration, and burnin at 20,000 iterations at which point the rate of deviation was below 0.01 . We used a uniform prior on topologies and exponential priors (10) on branch lengths. Branch lengths represent the number of expected substitutions (Fig. 1). The mitochondrial genes chosen for our reconstruction have been previously successfully used to build a robust phylogeny of 49 Tanganyika cichlid species (Salzburger et al. 2002) and the phylogenetic relationships in our tree are in accord with those presented by Salzburger et al. (2002).

BayesTraits (see below) can be applied to a sample of phylogenetic trees such that evolutionary models and hypotheses are tested taking phylogenetic uncertainty into account. Hence, we created a sample of 500 alternative phylogenetic trees for the 33 species included in the analyses by sampling 250 trees from each of the two independent runs of the MrBayes analysis after burnin, sampling from iteration 20,000 to iteration 3,000,000. Trees were sampled at intervals of 11,900 trees to minimize autocorrelation between successive iterations.

\section{COMPARATIVE ANALYSES}

The correlations between sexual selection and parental care type, sexual selection and female investment, and parental care type and female investment were analyzed using the Pagel 94 analysis for bivariate correlations as implemented in Mesquite (Maddison and Maddison 2007) using the consensus phylogeny obtained from MrBayes. The single polytomy in the consensus tree was resolved with a branch length of 0.0 in Mesquite. Furthermore, we analyzed the correlation between form of care (mouthbrooding or substrate guarding) and sexual selection, and form of care and female investment. The Pagel 94 analysis is based on a maximumlikelihood approach and estimates rates of change between two binary characters across the phylogeny without requiring reconstruction of ancestral states. The analysis compares the log likelihood of a model of evolution in which the two traits evolve independently across the phylogeny with the log likelihood of a model enforcing correlated evolution of the traits (Pagel 1994). The $P$ value of the difference between the two models of evolution can be obtained by applying a likelihoods ratio test: 2(loglikelihood [dependent model] - loglikelihood [independent model]), which has been shown to approximate a chi-square distribution with 4 degrees of freedom (Pagel 1994). In Results we show the value of the log-likelihood difference (likelihood difference) 


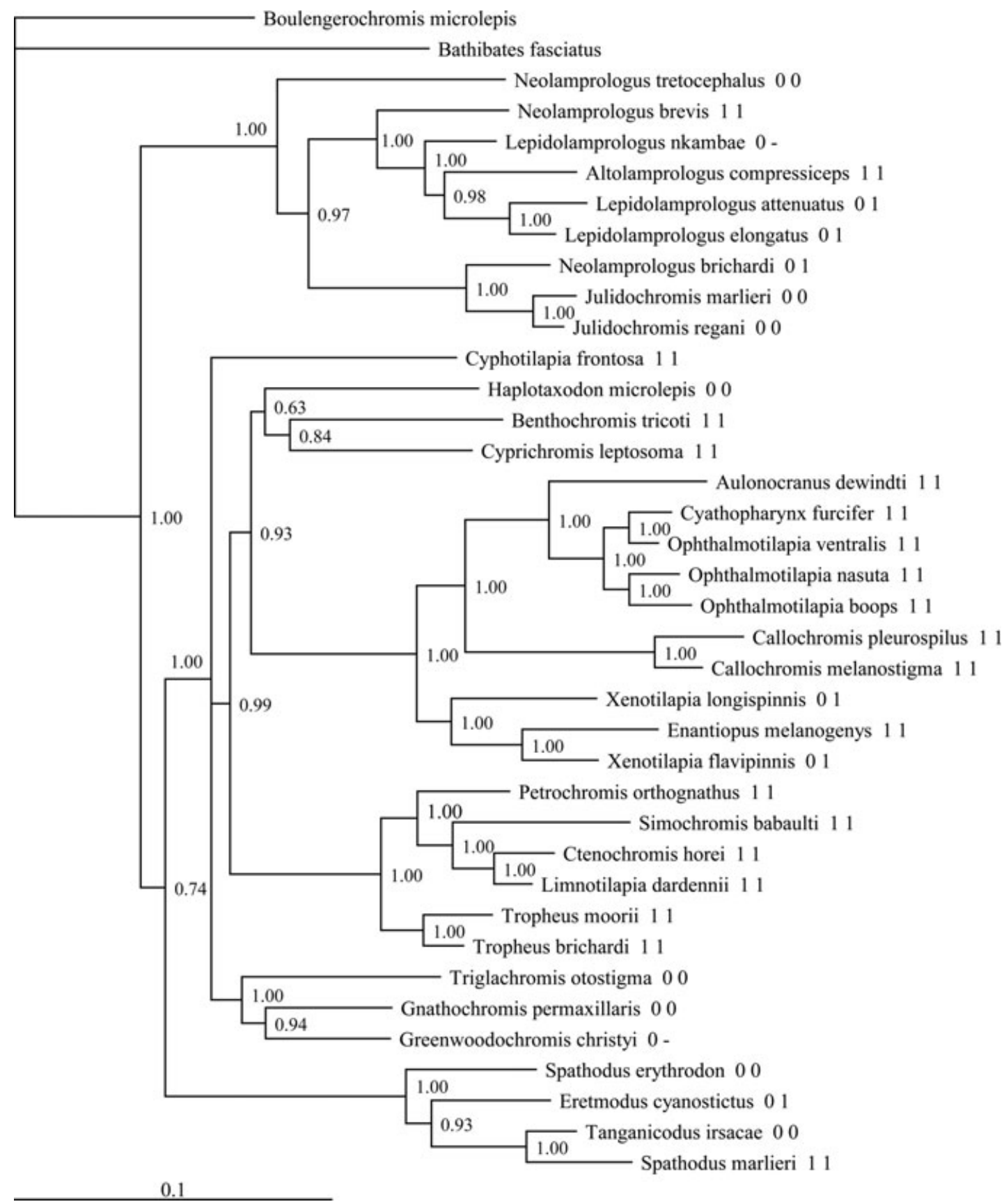

Figure 1. Consensus phylogenetic tree of 36 Tanganyikan cichlid species based on ND2, cyt $b$, and mitochondrial control region genes, with Boulengerochromis microlepis and Bathybates fasciatus set as the outgroup. Branches are drawn proportional to the number of expected substitutions. Numbers within the tree represent Bayesian posterior probabilities. Form of care $(0=b i p a r e n t a l, 1=$ female only) and intensity of sexual selection ( $0=$ moderate, $1=$ intense) are also shown for each of the species, dashes represent missing data.

between the correlated and independent models of evolution and the $P$ value. Because Pagel 94 cannot deal with missing data, for the analyses involving female investment we cropped the 36 species consensus tree to include only the 22 species for which we had data on female investment. For the correlation analyses between sexual selection and parental care and form of care and sexual selection we cropped the consensus tree to include only the 34 species for which we had data on sexual selection.
Finally we used the program BayesTraits (Pagel and Meade 2006), which combines Bayesian and maximum likelihood based approaches, to test the contingency of character evolution. Previous contingency analyses were limited to a maximum-likelihood approach (Thomas and Székely 2005; Krüger et al. 2007; Kolm et al. 2007). The method applies a continuous time Markov model of trait evolution that calculates the likelihood of discrete trait data under two models of evolution, one in which the traits are allowed 
to evolve independently of one another on a phylogenetic tree and one in which they evolve in a correlated fashion. The independent and dependent models can be compared by means of the harmonic mean of $\log$ likelihoods calculating 2(log[harmonic mean (dependent model)] - $\log [$ harmonic mean (independent model)]). For this comparison, a result greater than 2 is taken as positive evidence that the dependent model is favored, greater than 5 is strong and greater than 10 is very strong evidence (Pagel and Meade 2006). BayesTraits applies reversible-jump Markov chain Monte Carlo (RJ MCMC), which samples the posterior probability distributions of the parameter values of the model of trait evolution within a sample of phylogenetic trees that estimates the posterior probability of phylogenies. BayesTraits thus combines uncertainty about both the model of trait evolution and phylogenetic uncertainty by simultaneously sampling the posterior distribution of phylogenies and parameters of the model of trait evolution. The RJ MCMC method constructs a Markov chain that explores the universe of possible models, visiting them in direct proportion to their posterior probabilities (Pagel and Meade 2006). The parameters of the model of trait evolution are simply the values of the transition rates between the eight possible character states in a model of correlated evolution (the qs in Fig. 2A). The posterior probability distribution of the values of the parameters provides a means of analyzing the probability that the true value of the tran- sition parameter between two states of a trait is nonzero. This is simply calculated from the proportion of the evolutionary models visited by the Markov chain that assigned the parameter to a value of zero (A. Meade, pers. comm.). BayesTraits also calculates the posterior probability distribution for the possible ancestral states, thus allowing the researcher to infer the most likely ancestral state. The evolutionary path from ancestral state to derived state of two discrete traits can thus be inferred from the posterior probability distributions of the eight transition parameters. In our analysis the $\mathrm{PIH}$ predicts that the transition from, for example, an ancestral state of moderate sexual selection and biparental care to a derived state of intense sexual selection and female only care will necessarily involve first a transition in parental care type to female only care (Fig. 2B). Alternatively the SSH predicts that the same evolutionary path will instead involve first a transition in the intensity of sexual selection (Fig. 2B). Note that stating that a transition likely occurred before and another after makes no reference to one occurring earlier in the phylogenetic tree but rather reflects the contingency of trait evolution, that is that transitions in one trait depend on the state of the other trait.

Because our aim in the present study was to compare between the two alternative evolutionary pathways predicted by the $\mathrm{PIH}$ and SSH we first compared the independent and dependent models of trait evolution to confirm that, taking phylogenetic

A

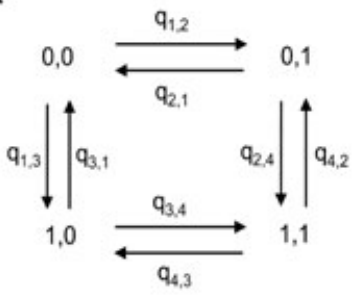

B

$\mathrm{PIH}$ prediction

\begin{tabular}{|c|}
\hline Biparental care \\
Intense sexual \\
selection
\end{tabular}

\begin{tabular}{|c|}
\hline Biparental care \\
Moderate sexual \\
selection
\end{tabular}

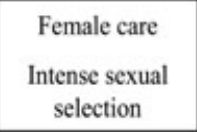

$\searrow$
Female care

Moderate sexual selection
SSH prediction

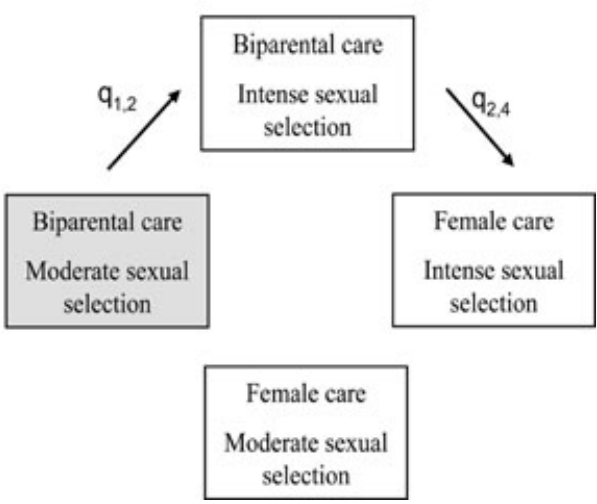

Figure 2. (A) Eight possible transitions among the four state combinations resulting from two binary traits. The subscripts identify the beginning and end states, respectively of each transition, where the values 1-4 correspond to the four state combinations. (B) Predicted evolutionary pathways from an inferred ancestral state (gray shaded box) of moderate sexual selection and biparental care to the derived state of intense sexual selection and female only care for the SSH and PIH. 
uncertainty into account, parental care and sexual selection were indeed correlated. Having confirmed the two traits were correlated we determined the most likely ancestral states of the traits from their posterior probability distributions. Finally, we analyzed the posterior probability distributions of the values of the transition parameters to determine the most likely evolutionary path from ancestral state to derived state. BayesTraits allows for missing data to be included in the analysis and these are treated as taking on both states. For the analyses we used a uniform prior for the independent model and an exponential hyperprior (0 100) for the model of dependent evolution as suggested by Pagel and Meade (2006). Repeating the analyses applying a uniform hyperprior for the dependent evolution model does not change the results. The analyses were run for 5,050,000 iterations with burnin at 50,000 iterations (Pagel and Meade Bayestraits manual) and sample frequency of 300 iterations. The harmonic means of the log likelihoods stabilize $\approx-29$ at just over $1,000,000$ iterations. The values of the $\mathrm{Z}$ scores and averages of the transition parameters do not change if calculated using 1,000,000th iteration as burnin.

\section{Results}

\section{BIVARIATE EVOLUTIONARY CORRELATIONS}

The analyses with Pagel 94, using the 34 species dataset, confirmed the correlation between sexual selection and parental care type (likelihood difference $=17.34, P=0.002$ ). Second, using the reduced dataset ( 22 species) we found that sexual selection and female investment were not correlated (likelihood difference $=0.74, P=0.94)$. Similarly, parental care type and female investment were not correlated (likelihood difference $=3.0, P=0.56$ ). Finally, the form of care (mouthbrooding or substrate guarding) was not associated with either sexual selection or female investment because the correlated evolution models did not differ significantly from the models of independent evolution (likelihood difference $=1.8, P=0.77$; likelihood difference $=1.12, P=$ 0.89 , respectively).

\section{CONTINGENCY ANALYSIS}

The results of the BayesTraits analysis confirmed the evolutionary correlation between parental care and intensity of sexual selection. The difference between the harmonic means of the independent and dependent models of trait evolution was 24.5. Because the harmonic means can be unstable the analysis was repeated several times and in all runs the magnitude of the difference between the harmonic means was highly similar, all $\approx 20$. Thus the results show very strong support for a model of correlated evolution. The posterior probability distributions of the ancestral states of the traits showed that the most likely ancestral state was biparental care and moderate sexual selection (average posterior probability $\pm \mathrm{SD}=60 \pm 15 \%$ ). This state was assigned a probability of 0 in as few as $0.3 \%$ of the over 16,600 iterations of the Markov chain that were sampled, whereas the ancestral state of female care and intense sexual selection was assigned a probability of 0 in $94 \%$ of the samples. Finally, the most likely evolutionary path from the ancestral to the derived state of the two traits of interest can be inferred from the posterior probabilities of the transition rate parameters (Fig. 3). The posterior distributions clearly show that the most likely path from the ancestral state of biparental care and moderate sexual selection to the derived state of female only care and intense sexual selection involved first a transition toward intense sexual selection $\left(q_{1,2}>0\right)$ followed by a transition toward female only care $\left(\mathrm{q}_{2,4}>0\right.$; Fig. 4$)$. Both transitions in this evolutionary path were assigned a value of zero in less than $5 \%$ of the sampled Markov chains (Fig. 3). The alternative route that involves first a transition toward female only care $\left(\mathrm{q}_{1,3} \approx 0\right)$ followed by a transition toward intense sexual selection $\left(\mathrm{q}_{3,4}>\right.$ 0 ) is not supported (Fig. 4). The two transitions involved in this alternative route were assigned a value of zero in 93 and $40 \%$ of the sampled Markov chains (Fig. 3). Thus our results support the $\mathrm{SSH}$ and suggest that evolutionary transitions in parental care are contingent on the intensity of sexual selection.

\section{Discussion}

Our results confirmed the existence of an evolutionary correlation between the intensity of sexual selection and parental care type. Intense sexual selection was associated with female only care, whereas moderate sexual selection was associated with biparental care. Furthermore, the results of the contingency analysis suggest that sexual selection determines parental care patterns: evolutionary transitions in parental care type were contingent on the intensity of sexual selection. The results show that the most likely evolutionary pathway from the ancestral state of moderate sexual selection and biparental care to intense sexual selection and female only care necessarily requires as a first step an increase in the intensity of sexual selection. It is worth pointing out that comparative analyses are correlative in nature and hence we cannot rule out that a third, unknown variable correlated to sexual selection and care type, is driving the relationship between these traits. However, our results provide no support for the PIH because in the Tanganyikan cichlids, parental care patterns do not determine sexual selection. Instead, our results are in accord with the SSH that suggests that rather than differences in relative parental investment leading to mating competition between members of the sex investing less, as suggested by Trivers (1972), sexual selection is the cause of relative differences between the sexes in parental investment (Wade 1979; Queller 1997).

Previous studies have suggested that biparental care is the ancestral state in Tanganyikan cichlids and that female only care is a derived state that has evolved from biparental care (Goodwin 


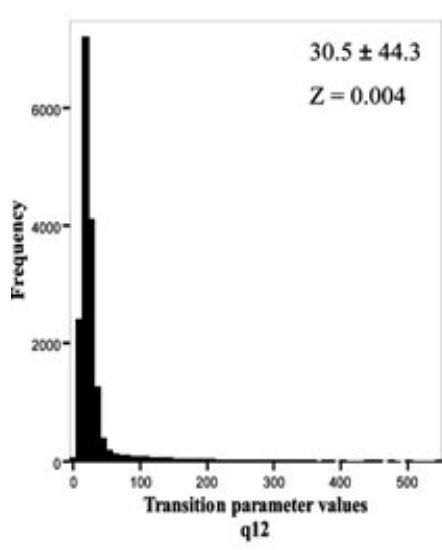

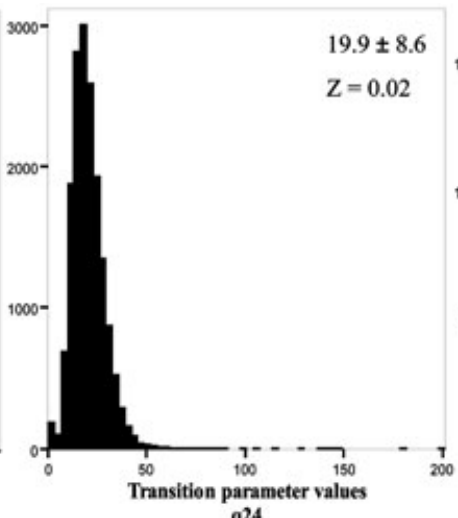

q24
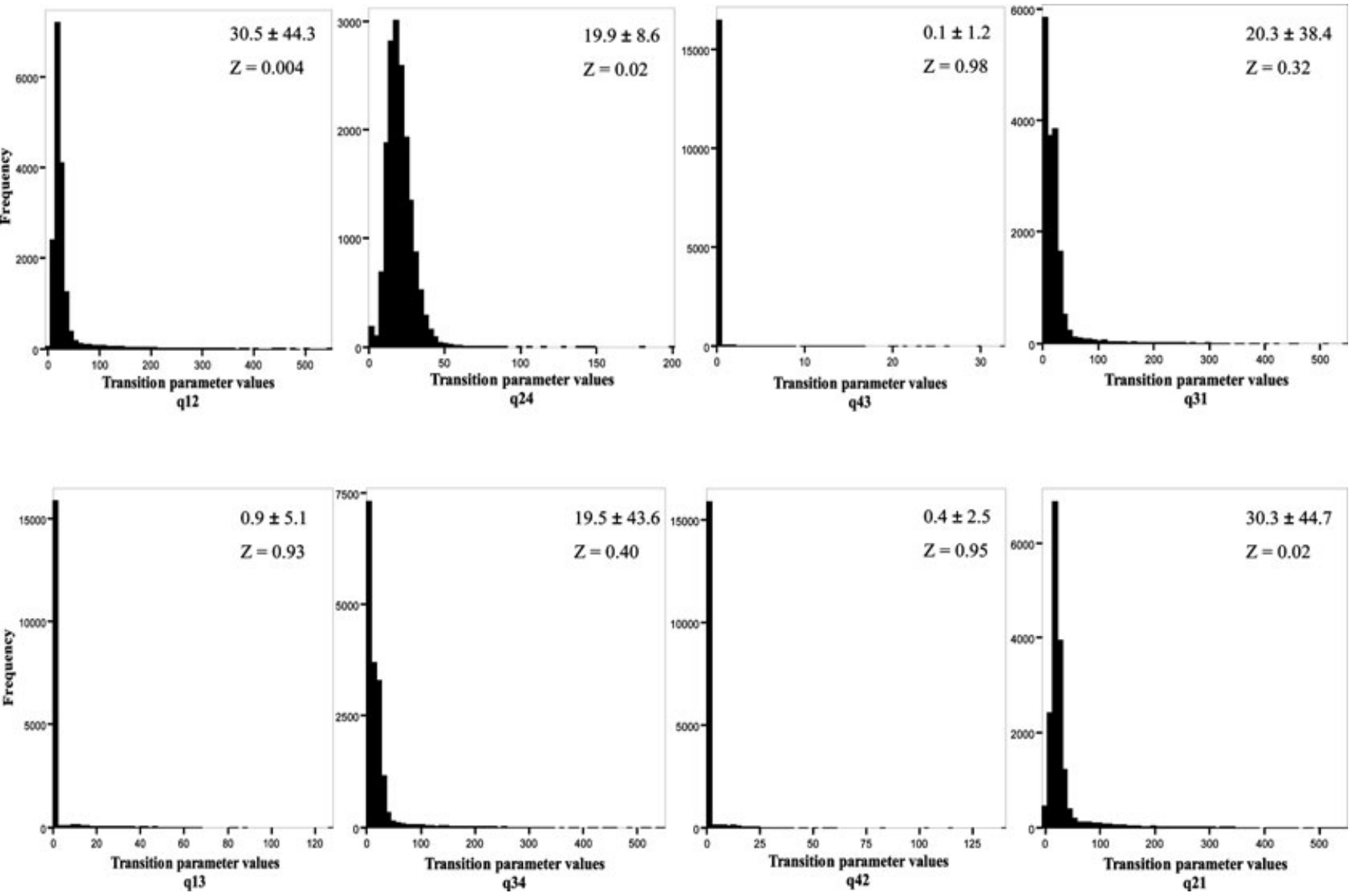

Figure 3. Posterior probability distributions of the values of the rate coefficients of the model of correlated evolution. $Z$ values present the proportion of the sampled runs from the Markov chain in which the parameter was assigned a value of 0 . Shown are the average and standard deviation of the parameter values from the sampled runs.

et al. 1998; Klett and Meyer 2002). Transitions from biparental to female only care were suggested to be primarily due to a reduction in care by males (Goodwin et al. 1998). Thus, our results are in accord with previously proposed patterns of evolution of parental care for the Tanganyika superflock. Similarly, in shorebirds, a decreased tendency for male care was attributed to possible benefits for males from additional mating opportunities (Reynolds and Székely 1997). Our results suggest that reversals in parental care type possibly have also taken place in the Tanganyikan cichlids. We found evidence for transitions from female care to biparental care (Fig. 4), but only in species with moderate sexual selection. Hence, this suggests that under low intensity of sexual selection there could be increased selection for male care (see Kokko and Jennions 2008). The positive transition rates leading away from a state of moderate sexual selection and female only care possibly arise because the model calculates the likelihood at internal nodes over all possible states. In this particular case, we could not find any extant species that presented moderate sexual selection and female only care, despite an intensive literature search and communication with Tanganyikan cichlid experts, and the pos- itive transition rates ensure that the model moves out of such a state quickly (Pagel and Meade 2006). The values of the transition parameters-and their Z scores - are, of course, influenced by the available data (as in any analysis). Absence of species presenting a specific combination of trait values makes it unlikely that the value of transitions leading to and away from this combination of states will be above zero and the $\mathrm{Z}$ scores low. The absence of species presenting moderate sexual selection and female care, in our study, probably results from selection acting against this combination of traits. Interestingly, our results also suggest that reversions from a derived state of intense sexual selection and female only care to the ancestral state of moderate sexual selection and biparental care are unlikely. Thus, in Tanganyikan cichlids, it appears there is no turning back from a state of intense sexual selection and female only care. It is possible that the intense sexual selection acting on males, which appears to be at the origin of the divergence in sex roles (Kokko and Jennions in press), possibly coupled with reduced certainty of paternity for males (at least in nonterritorial species) may also hinder a reversion toward biparental care and moderate sexual selection. 


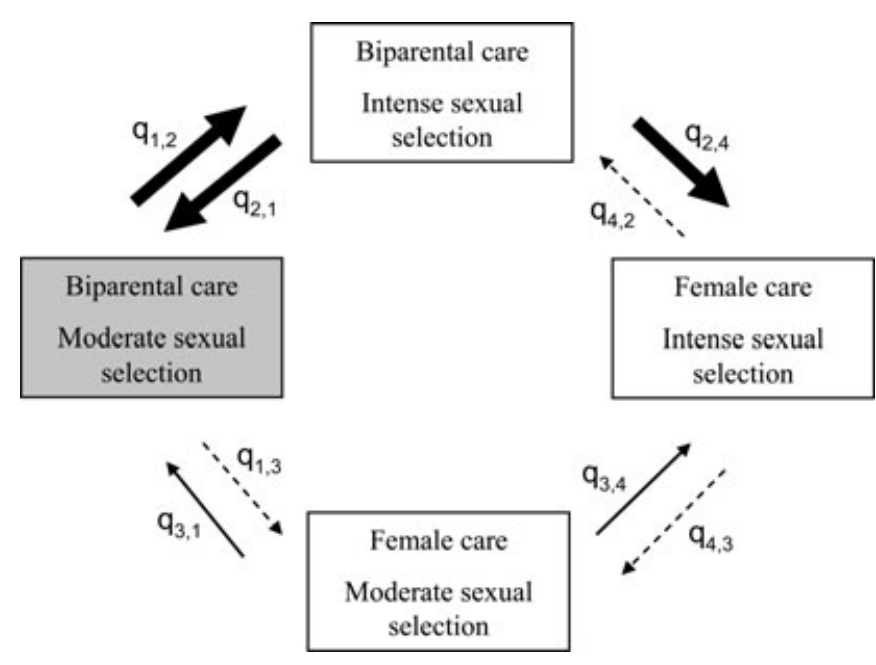

Figure 4. Flow diagram showing the most probable evolutionary pathway from the ancestral state of biparental care and moderate sexual selection (gray shaded box) to the derived state of female care and intense sexual selection. Thick black arrows highlight transitions that have a low posterior probability of being zero $(<5 \%)$, thin arrows denote transitions that a higher probability of being zero (31-40\%), and dashed arrows denote transitions that have a very high posterior probability of being zero (> $90 \%$ ).

Female investment was not significantly correlated with the intensity of sexual selection or with parental care type, which suggests that whether females lay relatively large or small clutches has no influence on a male's decision to invest in parental care or abandon the female to compete for other mating opportunities. We used clutch size and not egg size in our measure of female investment because a previous study showed that the size of the clutch is the variable most closely related to the duration of parental care, a pattern that is apparently driven by the duration of posthatch care in cichlids (Kolm et al. 2006). Form of care (mouthbrooding or substrate guarding) was not significantly correlated with the intensity of sexual selection or with female investment, thus we can discount possible confounding effects of whether the species is mouthbrooding or substrate guarding. A previous study suggested that biparental care in mouthbrooding cichlids was associated with large clutches that could not fit into the female's mouth requiring the assistance of the male for brooding (reviewed in Clutton-Brock 1991). Our results do not support this suggestion as there was no association between female investment and parental care type, or between female investment and form of care.

What selective forces might lead to males who are under intense sexual selection deserting the caring female, as suggested by our results? This is by no means self explanatory because male desertion is likely to lead to a male-biased operational sex ratio that should result in decreased expected fitness returns for abandoning males. However, as pointed out by Jennions and Kokko
(2008), and in line with the SSH, not all males encounter the alternative of either abandoning a female or contributing to parental care but only a subset of males who have proved successful in competing for or attracting females. An increase in the intensity of sexual selection acting on males might lead to increased variance in reproductive success if some males are unsuccessful in reproducing, be it as a result of being outcompeted by other males or being shunned by females. As pointed out by Kokko and Jennions (2008) in the context of parental investment evolution, the relevant measure of sexual selection is how much it elevates the future expected mating rate of individuals who have already mated. Although this scenario is proposed for the system under study, it could work equally well independently of whether sexual selection is acting on male-male competition or through mate choice. Therefore, we suggest that the SSH could be a general force behind the common pattern of caring females and competing males across taxa.

The Lamprologini tribe of Tanganyikan cichlids provides a nice example of how the environment can influence sexual selection and parental investment patterns (Emlen and Oring 1977). Species in this tribe have been categorized as permanently or temporarily haremic, bigamous, or monogamous (Gashagaza 1991). Males generally defend territories with suitable spawning sites (although in L. attenuatus the territories are not clearly defined), however the difference lies in the number of spawning sites within a male's territory. In some species (e.g., Altolamprologus compressiceps, Neolamprologus fasciatus and $N$. furcifer) males defend territories with several spawning sites, the mating system is polygynous and males provide no parental care. At the other extreme in monogamous species such as Neolamprlogus toae and $N$. tretocephalus males defend territories with a single spawning site and the pairbond can be long lasting with some pairs observed to spawn again following independence of their brood (Gashagaza 1991). Within this continuum of variation are $L$. attenuatus, L. elongatus, and N. brichardi, in which the territories of some males include only one spawning site whereas those of other males include two sites and there is a high prevalence of bigamy. All three species present biparental care and bigamous males apparently divide their investment between the two broods (Gashagaza 1991; Yanagisawa et al. 1997). Interestingly, the extent of SSD increases with the degree of polyandry: in the two monogamous species there is slight SSD, males being about $9 \%$ larger, in the two bigamous species males are $\approx 30 \%$ larger and in polyandrous species males are on average $41 \%$ larger than the female (Gashagaza 1991). Hence, the three states shown to be involved in the evolutionary path from ancestral to derived state in Tanganyikan cichlids are represented in a contemporary continuum of variation within the Lamprologini tribe: biparental species with moderate SSD, biparental species with marked SSD, and species with female only care and pronounced SSD. Note 
that territoriality has been shown to be correlated with paternal care in ray-finned fishes (Ah-King et al. 2005), and a directional phylogenetic analysis suggested that the evolution of territoriality tended to precede the evolution of male parental care (Ah-King et al. 2005). However, in Tanganyikan cichlids male territoriality appears rather to be associated with biparental or female only care.

If the $\mathrm{SSH}$ is a general phenomenon, it could provide an explanation for cases in which males are under stronger sexual selection than females even though parental care is provided exclusively by males, as occurs in most fish species with parental care (Baylis 1981; Clutton-Brock and Parker 1992; Andersson 1994). In these cases, there is no evidence for a trade-off between parental care and mate searching (Jennions and Kokko, in press), as males may rapidly switch from caring for eggs and courting females and females preferentially mate with males that provide more or higher quality parental care (Östlund and Ahnesjö 1998; Mank et al. 2005; Lindström et al. 2006). Thus, male care can in some cases be even sexually selected (Ah-King et al. 2005; Kolm and Ahnesjö 2005; Mank et al. 2005).

As mentioned above, our results suggest that in Tanganyikan cichlids, evolutionary transitions could potentially also have included a transition from female only care to biparental care in species already presenting moderate sexual selection. Under what circumstances might selection thus favor males providing parental care? Because a male's reproductive success is highly dependent on the competitive investment of other males, a considerable investment that is still below that of other competitors may result in null reproductive success (Andersson 1994). As mating competition intensifies, the costs of becoming a successful competitor will also increase to the point where the expected fitness benefits associated with polygyny might not offset the costs. Such circumstances could relax sexual selection acting on males. Moreover, Queller (1997) suggested selection would not favor males investing in parental care because males generally have less certainty of paternity when compared to females. However, a recent model has shown that fitness gains through male care of offspring are not intrinsically less valuable than those through desertion (Fromhage et al. 2007). As previously noted, territoriality has been shown to be correlated with paternal care in fish (Ah-King et al. 2005), and caring males may be in a better position to defend/ensure their paternity, thus territoriality could lead to male investment in parental care as a result of increased certainty of paternity (Kvarnemo 2006). Changes in the environment's potential for polygamy (Emlen and Oring 1977) or demographic population parameters for example influencing the sex ratio could also favor biparental care (Kvarnemo and Ahnesjö 1996). An experimental study with a Tanganyika biparental mouthbrooding cichlid (E. cyanosticus) showed that when the adult sex ratio is female biased males tend to reduce their investment in the brood and conflict about the amount of care provided by each sex emerges (Grüter and Taborsky 2005). Thus, the SSH highlights the possibility of an ecogenetic feedback loop (Kokko and López-Sepulcre 2007), as environmental or population parameters may influence the mating system and thus the intensity of sexual selection.

In conclusion, our results show that in Tanganyikan cichlids, evolutionary transitions in parental care type are contingent on the intensity of sexual selection (Fig. 4). Moreover, parental care patterns were not affected by female investment or form of care. Our results thus provide the first empirical support for the SSH in showing that sexual selection can govern, rather than be a consequence of patterns of parental investment. We suggest future empirical studies should test the generality of these results across different taxa as well as for different measures of the level of sexual selection. Experimental tests of the SSH are also an important direction for future studies: for instance, does parental investment decrease as the competitiveness or attractiveness of a male increase?

\section{ACKNOWLEDGMENTS}

This work was funded by the Wenner-Grens Foundations and the Swedish Research Council. AGV was supported by a Wenner-Grens Foundations post-doctoral stipend. JLF was supported by a Natural Sciences and Engineering Research Council of Canada (NSERC) postgraduate scholarship. R. Santos Gally and S. Castroviejo Fisher kindly assisted with phylogeny construction. I. Ahnesjö provided valuable comments on early drafts. A. Mooers and two anonymous reviewers provided valuable comments that improved the manuscript.

\section{LITERATURE CITED}

Ah-King, M., C. Kvarnemo, and B. S. Tullberg. 2005. The influence of territoriality and mating system on the evolution of male care: a phylogenetic study on fish. J. Evol. Biol. 18:371-382.

Andersson, M. 1994. Sexual selection. Princeton Univ. Press, Princeton, NJ.

Balshine-Earn, S., and D. J. D. Earn. 1998. On the evolutionary pathway of parental care in mouth-brooding cichlid fish. Proc. R. Soc. Lond. B 265:2217-2222.

Barlow, G. W. 2000. The cichlid fishes. Perseus Publishing, Cambridge, MA.

Barta, Z., A. I. Houston, J. M. McNamara, and T. Székely. 2002. Sexual conflict about parental care: the role of reserves. Am. Nat. 159:687705.

Bateman, A. J. 1948. Intrasexual selection in Drosophila. Heredity 2:349-368. Baylis, J. R. 1981. The evolution of parental care in fishes, with reference to Darwin's rule of male sexual selection. Env. Biol. Fish. 6:223-251.

Clutton-Brock, T. H. 1991. The evolution of parental care. Princeton Univ. Press, Princeton NJ.

Clutton-Brock, T. H., and G. A. Parker. 1992. Potential reproductive rates and the operation of sexual selection. Q. Rev. Biol. 67:437-456.

Emlen, S. T., and L. W. Oring. 1977. Ecology, sexual selection, and the evolution of mating systems. Science 197:215-223.

Erlandsson, A., and A. J. Ribbink. 1997. Patterns of sexual size dimorphism in African cichlid fishes. S. Afr. J. Sci. 93:498-508.

Fairbairn, D. J. 2007. The enigma of sexual size dimorphism. Pp. 1-10 in D. J. Fairbairn, W. U. Blanckenhorn, and T. Székely, eds. Sex, size, and 
gender roles: evolutionary studies of sexual size dimorphism. Oxford Univ. Press, Oxford.

Freckleton, R. P. 2002. On the misuse of residuals in ecology: regression of residuals vs. multiple regression. J. Anim. Ecol. 71:542-545.

Fromhage, L., J. McNamara, and A. I. Houston. 2007. Stability and value of male care for offspring: is it worth only half the trouble? Biol. Lett. 3:234-236.

Gashagaza, M. M. 1991. Diversity of breeding habitats in Lamprologine cichlids in Lake Tanganyika. Physiol. Ecol. Japan 28:29-65.

Goodwin, N. B., S. Balshine-Earn, and J. D. Reynolds. 1998. Evolutionary transitions in parental care in cichlid fish. Proc. R. Soc. Lond. B 265:2265-2272.

Grüter, C., and B. Taborsky. 2005. Sex ratio and the sexual conflict about brood care in a bi-parental mouthbrooder. Behav. Ecol. Sociobiol. 58:44-52.

Harvey, P. H., and M. D. Pagel. 1991. The comparative method in evolutionary biology. Oxford Univ. Press, Oxford, UK.

Houston, A. I., and J. M. McNamara. 2005. John Maynard Smith and the importance of consistency in evolutionary game theory. Biol. Philos. 20:933-950.

Huelsenbeck, J. P., F. Ronquist, R. Nielsen, and J. P. Bollback. 2001. Bayesian inference of phylogeny and its impact on evolutionary biology. Science 294:2310-2314.

Jennions, M. D., and H. Kokko. in press. Sexual selection. In D. F. Westneat and C. W. Fox, eds. Evolutionary Behavioral Ecology. Oxford Univ. Press, Oxford.

Klett, V., and A. Meyer. 2002. What, if anything is a Tilapia? Mitochondrial ND2 phylogeny of Tilapiines and the evolution of parental care systems in the African cichlid fishes. Mol. Biol. Evol. 19:865-883.

Kokko, H., and M. Jennions. 2003. It takes two to tango. Trends Ecol. Evol. 18:103-104.

- 2008. Parental investment, sexual selection and sex ratios. J. Evol. Biol. doi:10.1111/j.1420-9101.2008.01540.x

Kokko, H., and A. López-Sepulcre. 2007. The ecogenetic link between demography and evolution: can we bridge the gap between theory and data? Ecol. Lett. 10:773-782.

Kolm, N., and I. Ahnesjö. 2005. Do egg size and parental care co-evolve in fishes? J. Fish Biol. 66:1499-1515.

Kolm, N., N. B. Goodwin, S. Balshine, and J. D. Reynolds. 2006. Life history evolution in cichlids 1: revisiting the evolution of life histories in relation to parental care. J. Evol. Biol. 19:66-75.

Kolm, N., R. W. Stein, A. Ø. Mooers, J. J. Verspoor, and E. J. A. Cunningham. 2007. Can sexual selection drive female life histories? A comparative study on Galliform birds. J. Evol. Biol. 20:627-638.

Konings, A. 1998. Tanganyika cichlids in their natural habitat. Cichlid Press, El Paso.

- 2005. Back to nature guide to Tanganyika cichlids, 2nd edn. Cichlid Press, El Paso

Krüger, O., N. B. Davies, and M. D. Sorenson. 2007. The evolution of sexual dimorphism in parasitic cuckoos: sexual selection or coevolution? Proc. R. Soc. Lond. B 274:1553-1560.

Kuwamura, T. 1997. The evolution of parental care and mating systems among Tanganyikan cichlids. Pp. 59-86 in H. Kawanabe, M. Hori, and M. Nagoshi, eds. Fish communities in Lake Tanganyika. Kyoto Univ. Press, Kyoto.

Kvarnemo, C. 2006. Evolution and maintenance of male care: is increased paternity a neglected benefit of care? Behav. Ecol. 17:144-148.

Kvarnemo, C., and I. Anhesjö. 1996. The dynamics of operational sex ratios and competition for mates. Trends Ecol. Evol. 11:404-408.
Lindström, K., C. M. St. Mary, and C. Pampoulie. 2006. Sexual selection for male parental care in the sand goby, Pomatoschistus minutus. Behav. Ecol. Sociobiol. 60:46-51.

Maddison, W. P., and D. R. Maddison. 2007. Mesquite: a modular system for evolutionary analysis. Version 2.0. http://mesquteproject.org.

Mank, J. E., D. E. L. Pomislow, and J. C. Avise. 2005. Phylogenetic perspectives in the evolution of parental care in ray-finned fishes. Evolution 59:1570-1578.

Östlund, S., and I. Ahnesjö. 1998. Female fifteen-spined sticklebacks prefer better fathers. Anim. Behav. 56:1177-1183

Pagel, M. D. 1994. Detecting correlated evolution on phylogenies: a general method for the comparative analysis of discrete characters. Proc. R. Soc. Lond. B 255:37-45.

. 1999. Inferring the historical patterns of biological evolution. Nature 401:877-884.

Pagel, M. D., and A. Meade. 2006. Bayesian analysis of correlated evolution of discrete characters by reversible-jump Markov chain Monte Carlo. Am. Nat. 6:808-825.

Parker, G. A., and L. W. Simmons. 1996. Parental investment and the control of sexual selection: predicting the direction of sexual competition. Proc. R. Soc. Lond. B 263:315-321.

Queller, D. C., 1997. Why do females care more than males? Proc. R. Soc. Lond. B 264:1555-1557.

Reynolds, J. D., and T. Székely. 1997. The evolution of parental care in shorebirds: life histories, ecology, and sexual selection. Behav. Ecol. 8:126-134.

Ronquist, F., and J. P. Huelsenbeck. 2003. MrBayes 3: Bayesian phylogenetic inference under mixed models. Bioinformatics 19:15721574 .

Salzburger, W., A. Meyer, S. Baric, E. Verheyen, and C. Sturmbauer. 2002. Phylogeny of the Lake Tanganyika cichlid species flock and its relationship to the central and east African haplochromine cichlid fish faunas. Syst. Biol. 51:113-135.

Seehausen, O., P. J. Mayhew, and J. J. M. van Alphen. 1999. Evolution of colour patterns in East African cichlid fish. J. Evol. Biol. 12:514 534.

Tallamy, D. W. 2000. Sexual selection and the evolution of exclusive paternal care in arthropods. Anim. Behav. 60:559-567.

Thomas, G. H., and T. Székely. 2005. Evolutionary pathways in shorebird breeding systems: sexual conflict, parental care, and chick development. Evolution 59:2222-2230.

Trivers, R. L. 1972. Parental investment and sexual selection. Pp. 136-179 in E. B. G. Campbell, ed. Sexual selection and the descent of man. Aldine, Chicago.

Wade, M. J. 1979. Sexual selection and variance in reproductive success. Am. Nat. 114:742-747.

Wade, M. J., and S. M. Shuster. 2002. The evolution of parental care in the context of sexual selection: a critical reassessment of parental investment theory. Am. Nat. 160:285-292.

Westneat, D. F., and R. C. Sargent. 1996. Sex and parenting: the effects of sexual conflict and parentage on parental strategies. Trends Ecol. Evol. 11:87-91.

Yanagisawa, Y., H. Ochi, and M. M. Gashagaza. 1997. Habitat use in cichlid fishes for breeding. In H. Kawanabe, M. Hori, and M. Nagoshi, eds. Fish communities in Lake Tanganyika. Kyoto Univ. Press, Kyoto.

Associate Editor: A. Mooers 


\section{Supplementary Material}

The following supplementary material is available for this article:

Appendix S1. Accession numbers for the three mitochondrial genes used to construct the phylogeny.

Appendix S2. Values for parental care ( 0 biparental, 1 female only), intensity of sexual selection ( 0 moderate, 1 intense), mating system (0 monogamous, 1 nonmonogamous), sexual dimorphism ( 0 males less than $5 \%>$ females, 1 males $5 \%>$ females) and sexual dichromatism ( 0 monochromatic, 1 dichromatic) used in the comparative analyses.

Appendix S3. Measures of sexual dimorphism (male SL/female SL) and data sources for all variables included in the phylogenetic comparative analysis.

This material is available as part of the online article from:

http://www.blackwell-synergy.com/doi/abs/10.1111/j.1558-5646.2008.00426.x

(This link will take you to the article abstract).

Please note: Blackwell Publishing is not responsible for the content or functionality of any supplementary materials supplied by the authors. Any queries (other than missing material) should be directed to the corresponding author for the article. 